Center for Health Law and Policy Innovation, Harvard Law School, Harvard University, Cambridge, MA, USA

2 Division of General Medicine and Clinical Epidemiology, Department of Medicine, University of North Carolina at Chapel Hill School of Medicine, Chapel Hill, NC, USA

3 Cecil G Sheps Center for Health Services Research, University of North Carolina at Chapel Hill, Chapel Hill, NC, USA

4 Section of General Internal Medicine George Washington University School of Medicine and Health Sciences, George Washington University Culinary Medicine Program, George Washington University, Washington, DC, USA

5 Department of Community Health Sciences, Cumming School of Medicine, University of Calgary, Calgary, AB, Canada

6 Friedman School of Nutrition Science and Policy, Tufts University, Boston, MA, USA

Correspondence to: S Downer sdowner@law.harvard.edu

Cite this as: BMJ2020;369:m2482 http://dx.doi.org/10.1136/bmj.m2482 Published: 29 June 2020

FOOD FOR THOUGHT 2020

\title{
Food is medicine: actions to integrate food and nutrition into healthcare
}

\section{Sarah Downer and colleagues review new efforts to incorporate food and nutrition into prevention,} management, and treatment of diet related disease in healthcare systems

Sarah Downer, ${ }^{1}$ Seth A Berkowitz, ${ }^{2,3}$ Timothy S Harlan, ${ }^{4}$ Dana Lee Olstad, ${ }^{5}$ Dariush Mozaffarian ${ }^{6}$

\section{Key messages}

- In the face of the global epidemic of diet related chronic disease, there is increased experimentation with the use of "food is medicine" interventions to prevent, manage, and treat illness

- Interventions used with increasing frequency in the US and piloted to some extent in other countries include medically tailored meals, medically tailored groceries, and produce prescription programmes

- Scaled integration of these and other emerging nutrition interventions into healthcare would require significant investment in rigorous research to test different approaches and address knowledge gaps

- Clinicians need more and better education and training on the appropriateness and use of these interventions

- We also need to identify sustained funding streams to ensure equitable access and availability for patients

A global epidemic of diet related chronic disease has prompted experimentation using food as a formal part of patient care and treatment. One of every five deaths across the globe is attributable to suboptimal diet, more than any other risk factor including tobacco. ${ }^{1}$ Individual interactions with the healthcare system are an important opportunity to offer evidence based food and nutrition interventions. An emerging but compelling body of research indicates that such interventions delivered in the healthcare system might be associated with improved health outcomes and reduced healthcare usage and costs. ${ }^{2-10}$ These data point to the potential for food and nutrition interventions to play a prominent role in the prevention, management, treatment, and even in some cases reversal of disease. ${ }^{11}$ When broadly deployed, interventions that are effective for individual patients have the potential to affect population health and shape broader food and health policy reform. Realisation of health benefits is, however, hampered by lack of investment in research, low levels of clinician nutrition knowledge and awareness of interventions, and narrow access to appropriate services and programmes. Tackling each of these challenges is critical to achieving a healthcare system in which nutrition and food are a routine part of evidence based disease prevention and treatment.

We argue for increased integration of specific food and nutrition interventions in-or closely coordinated with-the healthcare system, an initiative often known as "food is medicine." We focus on novel interventions such as medically tailored meals and prescriptions for produce that incorporate food strategies to improve health in the structure and funding of the healthcare system rather than traditional medical nutrition interventions such as those that focus on vitamin or other nutrient supplements or medical foods.

\section{Food is medicine interventions}

Food is medicine interventions include medically tailored meals (also called therapeutic meals), medically tailored groceries (sometimes known as food "farmacies" or healthy food prescriptions), and produce prescriptions (table 1). They are typically directed by clinicians through the healthcare system, provided at no cost or very low cost to the patient, and funded by healthcare, government, or philanthropy. 


\begin{tabular}{|c|c|c|c|}
\hline Intervention & Definition & Target population & Research outcomes \\
\hline Medically tailored meals & \multicolumn{2}{|c|}{$\begin{array}{c}\text { Fully prepared meals designed by a professional Patients with complex medical conditions (such } \\
\text { based on an individual assessment. Typically as cancer, HIV, chronic heart failure) who are } \\
\text { includes individualised nutrition counselling unable to shop and preparing meals }\end{array}$} & 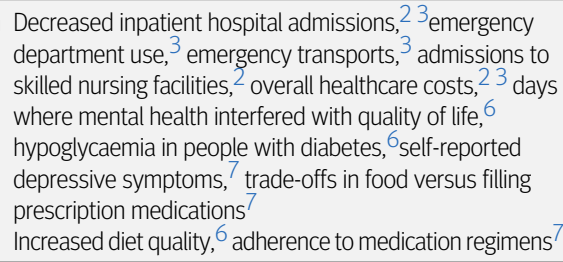 \\
\hline Medically tailored groceries & $\begin{array}{l}\text { Non-prepared grocery items selected by a } \\
\text { nutrition professional as part of a treatment } \\
\text { plan. Typically collected at a clinic or community } \\
\text { point and prepared at home. }\end{array}$ & $\begin{array}{l}\text { Patients with diet related chronic and acute } \\
\text { conditions (such as diabetes, cardiovascular } \\
\text { disease) who are also food insecure but able to } \\
\text { cook and prepare food at home }\end{array}$ & $\begin{array}{l}\text { Decreased } \mathrm{HbA1c} \text { in people with diabetes }{ }^{4} \text { and cost of care } \\
\text { where cost data were available } \\
\text { Increased medication adherence and fruit and vegetable } \\
\text { consumption }\end{array}$ \\
\hline Produce prescriptions & $\begin{array}{l}\text { Vouchers or debit cards for free or discounted } \\
\text { produce, distributed by healthcare providers. } \\
\text { Can be redeemed at various locations }\end{array}$ & $\begin{array}{l}\text { Patients who have or are at risk for diet related } \\
\text { chronic conditions (such as obesity or } \\
\text { prediabetes) and who are food insecure }\end{array}$ & 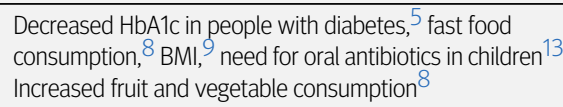 \\
\hline
\end{tabular}

Through a PubMed database search and by polling our network of international colleagues, we found 32 studies in the literature evaluating food is medicine interventions. Most of the examples we found were in the United States, with a few in other Western nations including Australia, Canada, and the United Kingdom. ${ }^{13^{-16}}$ We found none in Brazil, Finland, Germany, Ireland, or Mexico. Most interventions were dependent on philanthropic rather than organisational or institutional support. Reflecting the relative novelty of these efforts, data on the health impacts of food is medicine programmes were variably available in the peer reviewed literature.

These interventions vary widely in intensity and breadth of patient coverage. Medically tailored meals are the highest intensity intervention, necessary for a small but high needs group-those with complex medical conditions who are unable to shop or prepare meals. Over the past three years, the US has launched multiple large medically tailored meal projects for this population in their public insurance programmes, with additional use for elderly people proposed through legislation. ${ }^{17-19}$ The research on medically tailored meals cited in this article includes larger sample populations and more robust research designs (instrumental variable analyses, statistical matching, and a randomised crossover trial) than for the other interventions. ${ }^{2367}$ In a retrospective cohort study with 1020 participants, for example, receipt of medically tailored meals was associated with a $16 \%$ net reduction in overall healthcare costs, 49\% fewer inpatient hospital admissions, and $72 \%$ fewer admissions into skilled nursing facilities compared with the control group. ${ }^{2}$

Medically tailored groceries are appropriate for a broader range of patients-those with diet related chronic and acute conditions but who can cook and prepare food at home. Peer reviewed research on the impact of tailored packages of unprepared foods is scant, but co-location of food pantries and hospitals or health centres is increasingly common, along with ability to access this intervention at food banks. ${ }^{142021}$ Two randomised control trials are currently under way to assess recipient health impacts, one evaluating the impact of receiving nutritionally appropriate staple foods from a hospital located food pantry for people with diabetes and the second assessing home delivery of a medically tailored meal kit for low income pregnant women that meets certain diet related health eligibility criteria. ${ }^{22} 23$

In theory, produce prescriptions are appropriate for the broadest number of recipients, for both disease prevention and management. Several studies have explored the impact of these programmes on participant attitudes, behaviours, and consumption of fruits and vegetables, but research assessing clinical outcomes and claims data are relatively recent. Research on this intervention is trending in recent years towards more robust study designs with larger sample sizes. ${ }^{59}$ Modelling studies indicate that prescriptions for an array of healthful foods can be highly cost effective or even cost saving for the healthcare system when targeting key sociodemographic subgroups at highest risk, such as elderly people, adults with disabilities, and people with low income. ${ }^{10}$ Researchers in the US found, for example, that, over a lifetime, a 30\% subsidy incentive on fruits and vegetables would prevent 1.93 million cardiovascular disease events and save approximately \$4obn (32bn;

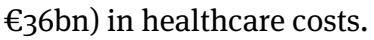

\section{Food insecurity}

We distinguish food is medicine interventions from programmes that respond to general food insecurity by being designed or administered with the express purpose of tackling health concerns. Food banks in the UK are an example of food insecurity response; access to food banks sometimes requires a referral from a healthcare provider, but the food received is not tailored, or not always appropriate, for people living with or at risk of specific health conditions. ${ }^{24}$ By contrast, at a preventive food pantry in a hospital in the US a hospital dietitian reviews the patient's medical record before selecting a mix of shelf stable and fresh foods that are nutritionally appropriate based on the individual's health profile and personal preferences. ${ }^{20}$

Given what we know about the impact of food insecurity on individual health and the healthcare system, ${ }^{25}{ }^{26}$ programmes like the UK's food bank voucher system, the US Supplemental Nutrition Assistance Program (formerly known as Food Stamps), and Brazil and Mexico's conditional cash transfer programmes are likely to provide some protection against adverse health outcomes. But the support they provide is not coordinated with the healthcare system, so specific health impacts are difficult to measure. Innovations that include clinical evaluations, clinical guidance, and financial support for food is medicine interventions in the healthcare system might be effective and expedient ways to improve both food insecurity and health, even if they do not comprehensively tackle the root structural causes of suboptimal diet.

In the US, government and private health insurers are adopting food and nutrition interventions in the hope of a return on their investment due to reductions in high expenditure healthcare claims. In light of emerging evidence, policy makers are experimenting with loosening the parameters of value based or capitated payment 
structures to allow public insurance money to be spent on food is medicine interventions. This flexibility can be expanded, administratively or through legislation, to all public health insurance programmes. However, large scale uptake of newly created flexibility to pay for food depends on confidence that food is medicine interventions are clinically effective and cost effective compared with other aspects of medical care.

\section{Benefits of food is medicine}

Referring patients to food is medicine interventions can change their ability to follow dietary recommendations, tackling several barriers to healthy eating, including the inability to afford or access recommended foods. ${ }^{2425-29}$ Providing food or food focused financial assistance can also alleviate budget constraints that prevent patients from affording medications and paying bills. ${ }^{28}$ Some food is medicine interventions model appropriate portion size and ingredient selection, enabling recipients to maintain more healthful diets past the intervention duration. ${ }^{28}$

Clinicians who refer patients to food is medicine interventions might also see better disease management and fewer admissions to hospital. . $-467^{-}$A patient with diabetes who typically runs out of food when monthly assistance is exhausted, for example, could be given anticipatory nutrition guidance and vouchers for supplemental food to avoid an episode of hypoglycaemia.

\section{Integration in healthcare}

Healthcare systems are a logical delivery or connection point for food is medicine interventions, but integration depends on many factors. We discuss the need for additional data on effectiveness of different food and nutrition interventions, increased clinician knowledge and familiarity, and sustainable funding.

\section{We need more data}

Most studies to date are quasi-experimental or small, short term pilot interventions. Key evidence gaps include the comparative efficacy of different interventions on physical, social, and mental health outcomes and healthcare utilisation, heterogeneity of treatment effects (which interventions work best for which groups, defined by both clinical and social circumstances), and the optimal intensity and duration of intervention needed for different situations. Given that the interventions might have effects throughout life, the appropriate timescale to assess benefits should also be considered.

Supportive evidence can be derived from careful modelling and microsimulation studies to forecast and compare dietary, health, and utilisation and cost benefits for different interventions and scenarios over the short and long term.

The promising findings observed in studies to date must be evaluated with larger implementation studies, including randomised trials with appropriate comparison groups. These might not always be feasible because nutrition research questions often involve long timeframes with treatments that might be difficult to standardise. Instead, quasi-experimental designs with low risk of bias can be used, when appropriate. These could include analyses of natural experiments, instrumental variable analyses, regression discontinuity studies, interrupted time series approaches, and difference-in-difference designs.

For food is medicine interventions to be most effective, the participant should be engaged and their experience evaluated. Qualitative research will better integrate individual perspectives into the design of food is medicine programmes and reveal how, why, and in what context they work best for participants. ${ }^{30}$ To tackle historical mistrust of the healthcare system and ensure cultural appropriateness of offerings, research to integrate food is medicine services into healthcare and bring them to scale should contemplate co-design of interventions with eligible participants and appropriate community based organisations. ${ }^{31}$

\section{Improving clinicians' knowledge}

Clinicians should be knowledgeable enough to recognise a patient's nutritional needs and understand the potential impact of available services, but this is not the case in many countries. In the US, for example, healthcare professionals (other than registered dietitians) receive very little or no food and nutrition education during training, with less than $1 \%$ of lecture hours spent on nutrition education in medical school. ${ }^{32}$ An assessment of medical nutrition education initiatives in six countries outside Europe and 15 in Europe found that "nutrition is insufficiently incorporated into medical education, regardless of country, setting, or year of medical education."33

Numerous recommendations have been made over the past 20 years to advance policies to increase nutrition education, but change remains elusive. ${ }^{34}$ Theories for the lack of progress include lack of leadership or faculty level nutrition champions at many medical schools, lack of compelling training opportunities (such as fellowships) focused on nutrition, and rapidly advancing nutrition science coupled with rampant public nutrition misinformation might have minimised the perceived credibility of nutrition science among physicians. ${ }^{34-37}$ Most physicians, however, recognise their lack of sufficient nutrition education, and would like more information to tackle this key driver of health. ${ }^{38}$

Nutrition training delivered across disciplines holds the promise of more effective patient nutrition education and treatment. ${ }^{39}$ In the US, UK, and Spain, “culinary medicine” movements are blending clinical medicine with individual nutrition education focused on the practical aspects of food preparation and cooking. This supports a healthcare professional's willingness and ability to recognise nutrition needs and provide appropriate and practical advice to patients..$^{40}{ }^{41}$ Clinicians should have familiarity with validated nutrition assessment tools, the range of available food is medicine interventions, and the systems and incentive structures that enable and encourage their use in clinical practice. Doctors should also understand the role of, and actively collaborate with, dietitians to appropriately treat people who are at nutritional risk. Increasing nutrition education among doctors might also encourage use of dietitians' expertise in patient care; rates of referral from physicians to dietitians are often quite low, even when diagnoses have a clear nutritional link. 42

Requiring comprehensive nutrition training as a component of healthcare clinician education will ensure equitable patient access to nutrition expertise across specialties and geographies. Ways to ensuring incorporation of nutrition into clinician curriculums include legislative mandates, making government funding for schools contingent upon such requirements, integration into accreditation standards, and inclusion of nutrition questions on board and other qualifying examinations. 43

\section{Sustainable funding in and out of healthcare}

The food is medicine interventions we found were largely funded by philanthropy and thus vulnerable to downturns in charitable giving and time limits on grants. The absence of sustainable funding mechanisms means that, in many locations, these services might simply not be available because health systems or community based organisations lack the resources. Thus, widespread implementation of food is medicine interventions, particularly those delivered 
through or with the healthcare system, requires financing models that consistently support these services. Sustainable support could be through public or private healthcare money or through other government funds.

In many healthcare systems, healthcare dollars pay for interventions such as enteral or intravenous feeding support and nutrition supplements for defined clinical deficiencies. But payment for food or meals is often allowed in only narrow circumstances-for example, for inpatients, people in assisted living facilities or nursing homes, or for specific nutritional items (as in the UK's prescription for gluten-free breads and mixes). ${ }^{44}$ This restrictive strategy might not be wise. In the US, an individual can receive seven months of medically tailored meals, nutrition counselling, and case management for the average cost of one inpatient hospital admission. ${ }^{45}{ }^{46}$ Because many of these interventions are provided in outpatient settings, trends towards shorter hospital stays would not decrease their efficacy and might make them even more important. If the provision of food was found to affect the rate of hospital admissions and other high cost services, ${ }^{23}$ health policy makers would have a powerful incentive to alter healthcare funding restrictions. Some US health system entities, including healthcare payers and provider organisations, are using recent changes to healthcare funding parameters to provide nutrition interventions to patients who meet certain criteria (box 1). ${ }^{17} 18$

\section{Box 1: Examples of integration of food in medicine interventions in healthcare in the US}

The states of Massachusetts ${ }^{17}$ and California ${ }^{18}$ are using food in medicine interventions with high risk populations. The government pays for these services through the healthcare system. The Massachusetts programme, launched in 2019, provides home delivered meals (medically tailored and non-medically tailored), groceries, assistance applying for non-healthcare nutrition programmes and legal advocacy for benefits, household supplies to meet dietary needs (cooking implements), nutrition skills development through education and cooking classes, and transportation to meet nutritional needs. To be eligible, patients must have one health needs based criterion (mental health condition, high risk pregnancy, complex health condition, has visited the emergency department more than twice in the past six months, has one or more limitations in activities of daily living) and one risk factor (homelessness, risk of homelessness, risk of nutritional deficiency or imbalance due to food insecurity). The programme measures the following outcomes: emergency department use, inpatient hospital admissions, overall healthcare expenditures, clinical outcomes, and the ability to live independently in the community. Results from the demonstration will be reported in 2022.

In addition to the food is medicine programmes funded through the healthcare system, the government has also appropriated millions of dollars of agriculture funding to establish produce prescription programmes in eight states around the country.

Payment mechanisms need not necessarily involve the healthcare system, as long as they are designed with health promotion in mind and are coordinated with interventions administered through the healthcare system. Health professionals in Alberta, Canada, for example, can confirm diagnosis of qualifying health conditions to enable social service recipients to receive an additional C $\$ 21-\mathrm{C} \$ 113$ a month in cash to subsidise the costs of recommended foods. ${ }^{15}$

When food is medicine interventions meet a standard of evidence that shows desired levels of impact on individual health outcomes and/or other desirable outcome measures, these services should be fully integrated into healthcare or other sustainable financing models. Reimbursement will support access to interventions, especially more complex services like medically tailored meals, by helping to create and sustain an infrastructure of organisations that can work with complicated and sensitive health information to deliver sophisticated interventions to anyone who meets eligibility criteria, whether they reside in urban or rural locations.

The food is medicine interventions reviewed in table 1 , administered in or closely coordinated with healthcare systems, are often enhanced and more targeted versions of services provided in anti-hunger programmes (see supplementary table online). These might include Germany's food bank system, which is entirely divorced from the healthcare system; universal school meals programmes (in Finland and other European countries); and services delivered through cash transfers conditioneal on receipt of certain healthcare services (such as those in Brazil and Mexico) or health diagnoses (in Canada). ${ }^{15} 47^{-50}$

\section{Future directions}

Integration of food is medicine interventions into healthcare depends in large part on new investment in research to add to the evidence base. Improved clinician training and referral capacity, together with increased financial support for interventions both in and outside the healthcare system, will help to ensure that patients are assessed and referred to appropriate interventions available in every community. Access to interventions will be supported by the proliferation of organisations and entities that are able to deliver a range of food and nutrition interventions, some of which are quite complex. In the US, for example, an increasing number of non-profit and for-profit entities are contracting with the healthcare system to provide these services, many for the first time.

The global pandemic of covid-19 has brought the fragility of food and healthcare systems across the globe into sharp relief, with skyrocketing rates of food insecurity and people with diet related illness struggling with increased barriers to accessing healthy food. ${ }^{25}{ }^{26}$ Healthcare systems that integrate food is medicine interventions will enable more resilient systemic responses to such crises. An integrated system will support an infrastructure of food is medicine providers and access pathways that can be used to immediately meet increased demand for healthy food support.

The consequences of poor health caused by poor diet affect many sectors (resulting in high healthcare spending that diverts funds from other policy priorities such as education and enhancing economic prosperity); clinicians working to advance integration have a compelling case to make to a diverse range of decision makers. As healthcare systems continue to evolve to tackle the global crisis of nutrition related disease, food is medicine interventions should be held to rigorous standards when decisions about implementation, coverage, and care are made. But they can no longer be categorically excluded as outside of or ancillary to healthcare delivery, as they have been in the past across many healthcare systems. Integration of food and nutrition interventions into healthcare holds significant promise for meeting immediate nutrition needs while working in harmony with broader, long term health and food system reforms.

Contributors and sources The expertise of the authors includes dietary patterns (DLO, TSH, SAB, DM), nutrition and health related public policy (SD, DLO, DM), clinician nutrition education and training (TSH, $\mathrm{DM})$, primary care and health related social needs interventions (SAB, DLO), and socioeconomic inequities in diet and health (all). SAB, DLO, TSH, and DM are clinicians. All authors contributed to drafting this manuscript, with SD taking a lead role. All authors gave intellectual input to improve the manuscript and have read and approved the final version. SD is guarantor.

Conflicts of Interest We have read and understood BMJ policy on declaration of interests and have the following interests to declare: SD reports organisational funding from the Rockefeller Foundation, the Laura and John Arnold Foundation, the MAC AIDS Fund, and the Bristol-Myers Squibb Foundation. She sits on the American Cancer Society's National Lung Cancer Roundtable (unpaid) and on the Advisory Board for the Aspen Institute Food and Society Program's food is medicine initiative. Funding for SAB's work on the studies cited in this article was provided by the National Institute of Diabetes and Digestive 
and Kidney Diseases of the National Institutes of Health under award number K23DK109200. The content is solely the responsibility of the authors and does not necessarily represent the official views of the National Institutes of Health. SAB has also received funding from the Aspen Institute, the US Department of Agriculture, the Robert Wood Johnson Foundation, and the Commonwealth Fund. He serves on the research advisory committee (unpaid) of the Social Intervention Research and Evaluation Network. TH reports past funding from the Robert Wood Johnson Foundation and the Humana Foundation; speaker fees from Optum, Omnia Education, beef.org, NACCME, DairyMAX; serves on the board of the Culinary Medicine Specialist Board; receives book royalties from Pritichett and Hull and Perseus; and is the owner and editor and chief of drgourmet.com and Dr Gourmet published materials. DLO reports research funding from the Canadian Institutes of Health Research, the Social Sciences and Humanities Research Council of Canada, the O'Brien Institute for Public Health, I Can For Kids Foundation, Alberta Innovates, Alberta Health Services, the Canadian Foundation for Dietetic Research, the Calgary Centre for Clinical Research, SecondBite, the University of Calgary, the Heart and Stroke Foundation of Canada, and the Libin Cardiovascular Research Institute of Alberta. DM reports research funding from the National Institutes of Health and the Gates Foundation; personal fees from GOED, Nutrition Impact, Bunge, Indigo Agriculture, Motif FoodWorks, Amarin, Acasti Pharma, Cleveland Clinic Foundation, America's Test Kitchen, and Danone; scientific advisory board, Brightseed, DayTwo, Elysium Health, Filtricine, HumanCo, and Tiny Organics; and chapter royalties from UpToDate.

Provenance and peer review: Commissioned; externally peer reviewed.

This article is part of series commissioned by The BMJ. Open access fees are paid by Swiss Re, which had no input into the commissioning or peer review of the articles. The BMJ thanks the series advisers, Nita Forouhi, Dariush Mozaffarian, and Anna Lartey for valuable advice and guiding selection of topics in the series.

This article draws from the law and policy expertise and research of so many, especially Kathryn Garfield, Kristin Sukys, Emily Broad Leib, and Robert Greenwald.

1 GBD 2017 Diet Collaborators. Health effects of dietary risks in 195 countries, 1990-2017: a systematic analysis for the Global Burden of Disease Study 2017. Lancet 2019;393:1958-72. doi: 10.1016/S0140-6736(19)30041-8 pmid: 30954305

2 Berkowitz SA, Terranova J, Randall L, Cranston K, Waters DB, Hsu J. Association between receipt of a medically tailored meal program and healthcare use. JAMA Intern Med 2019;179:786-93. doi: 10.1001/jamainternmed.2019.0198 pmid: 31009050

3 Berkowitz SA, Terranova J, Hill C, etal. Meal delivery programs reduce the use of costly healthcare in dually eligible Medicare and Medicaid beneficiaries. Health Aff 2018;34:535-42doi: 10.1377/hlthaff.2017.0999.

4 Seligman HK, Lyles C, Marshall MB, etal. A pilot food bank intervention featuring diabetes appropriate food improved glycemic control among clients in three states. Health Aff (Millwood) 2015;34:1956-63. doi: 10.1377/hlthaff.2015.0641 pmid: 26526255

5 Bryce R, Guajardo C, llarraza D, etal. Participation in a farmers' market fruit and vegetable prescription program at a federally qualified health center improves hemoglobin A1C in low income uncontrolled diabetics. Prev Med Rep 2017;7:176-9. doi: 10.1016/.jpmedr.2017.06.006 pmid: 28702315

6 Berkowitz SA, Delahanty LM, Terranova J, etal. Medically tailored meal delivery for diabetes individuals with food insecurity: a randomized cross-over trial. J Gen Intern Med 2019;34:396-404 doi: 10.1007/s11606-018-4716-z pmid: 30421335

7 Palar K, Napoles T, Hufstedler LL, etal. Comprehensive and medically appropriate food support is associated with improved HIV and diabetes health. J Urban Health 2017;94:87-99. doi: 10.1007/s11524-016-0129-7 pmid: 28097614

8 Trapl ES, Smith S, Joshi K, etal. Dietary impact of produce prescriptions for individuals with hypertension. Prev Chronic Dis 2018;15:. doi: 10.5888/pcd15.180301 pmid: 30447106

9 Cavanagh M, Jurkowski J, Bozlak C, Hastings J, Klein A. Veggie Rx: an outcome evaluation of a healthy food incentive programme. Public Health Nutr 2017;20:2636-41. doi: 10.1017/S1368980016002081 pmid: 27539192

10 Lee Y, Mozaffarian D, Sy S, etal. Cost-effectiveness of financial incentives for improving diet and health through Medicare and Medicaid: A microsimulation study. PLoS Med 2019;16: doi: 10.1371/journal.pmed.1002761 pmid: 30889188

11 Lean MEJ, Leslie WS, Barnes AC, etal. Durability of a primary care-led weight-management intervention for remission of type 2 diabetes: 2-year results of the DiRECT open-label, cluster-randomised trial. Lancet Diabetes Endocrinol 2019;7:344-55. doi: 10.1016/S2213-8587(19)30068-3. pmid: 30852132

12 Feinberg AT, Hess A, Passaretti M, Coolbaugh S, Lee TH. Prescribing food as a specialty drug. NEJM Catal, 2018, doi: 10.1056/CAT.18.0212.

13 Black AP, Vally H, Morris PS, etal. Health outcomes of a subsidised fruit and vegetable program for Aboriginal children in northern New South Wales. Med J Aust 2013;199:46-50. doi: 10.5694/mja13.10445 pmid: 23829264

14 Miller R. Filling 'prescriptions' for fruit and veggies blowing food bank's budget, CBC News, https://www.cbc.ca/news/canada/ottawa/prescriptions-for-healthy-food-blow-budget-1.5282589

15 Special Diets, Income \& Employment Supports Policy Manual, Alberta Government, http://www.humanservices.alberta.ca/AWonline/IS/4874.html.

16 Buyuktuncer Z, Kearney M, Ryan CL, Thurston M, Ellahi B. Fruit and vegetables on prescription: a brief intervention in primary care. J Hum Nutr Diet 2014;27(Suppl 2):186-93. doi: 10.1111/jhn.12109 pmid: 23651065
17 Massachusetts Executive Office of Health and Human Services. Performance year 3 (PY3) delivery system reform incentive payment (DSRIP) flexible services (FS) program guidance document for MassHealth accountable care organizations and MassHealth community partners. 2019. https://www.mass.gov/doc/flexible-services-guidance-document/download

18 California Department of Healthcare Services. Medically tailored meal pilot program. https://www.dhcs.ca.gov/services/ttc/Pages/Medically-Tailored-Meals-Pilot-Program.aspx

19 Medically-Tailored Home-Delivered Meal Demonstration Act of 2020. HR 6774. 116th US Congress. https://www.congress.gov/bill/116th-congress/house-

bill/6774?q=\%7B\%22search\%22\%3A\%5B\%22medically+tailored+meals\%22\%5D\%7D\&s=1\& $r=$

20 Preventive food pantry. Boston Medical Center. https://www.bmc.org/nourishing-our-community/preventive-food-pantry.

21 Fresh food farmacy https://www.geisinger.org/freshfoodfarmacy.

22 Hess A, Passaretti M, Coolbaugh S. Fresh food farmacy, a randomized control trial. Am J Health Promot 2019;33:830-2doi: 10.1177/0890117119845711d

23 Berger E. Local food bank expands innovative program to help expecting mothers. Alive 2020 Dec 28. https://alivemag.com/local-food-bank-expands-innovative-program-to-help-expecting mothers/

24 Wolfson JA, Ramsing R, Richardson CR, Palmer A. Barriers to healthy food access: Associations with household income and cooking behavior. Prev Med Rep 2019:13:298-305. doi: 10.1016/j.pmedr.2019.01.023. pmid: 30792944

25 Berkowitz SA, Basu S, Meigs JB, Seligman HK. Food insecurity and healthcare expenditures in the United States, 2011-2013. Health Serv Res 2018;53:1600-20doi: 10.1111/1475-6773.12730

26 Seligman HK, Jacobs EA, Lopez A, Tschann J, Fernandez A. Food insecurity and glycemic control among low-income patients with type 2 diabetes. Diabetes Care 2012;35:233-8doi: 10.2337/dc11-1627.

27 Fitzpatrick K, Harris C, Dawve G. Assessing US Food Insecurity in the United States during covid-19 pandemic. https://fulbright.uark.edu/departments/sociology/research-centers/community-familyinstitute/_resources/community-and-family-institute/revised-assessing-food-insecurity-brief.pdf

28 Berkowitz SA, Shahid NN, Terranova I, etal. "I was able to eat what I am supposed to eat”- - patient reflections on a medically-tailored meal intervention: a qualitative analysis. BMC Endocr Disord 2020;20:10. doi: 10.1186/s12902-020-0491-z. pmid: 31959176

29 COVID-19 will double number of people facing food crises unless swift action is taken, World Food Programme. https://www.wfp.org/news/covid-19-will-double-number-people-facing-foodcrises-unless-swift-action-taken

30 Patient-Centered Outcomes Research Institute. Methodology standards. 2019 Feb 26 https://www.pcori.org/research-results/about-our-research/research-methodology/pcorimethodology-standards\#QualitativeMethods.

31 Green T, Bonner A, Teleni L, etal. Use and reporting of experience-based codesign studies in the healthcare setting: a systematic review. BMJ Qual Saf 2020;29:64-76. doi: 10.1136/bmjqs-2019-009570 pmid: 31548278

32 Devries S, Dalen JE, Eisenberg DM, etal. A deficiency of nutrition education in medical training. Am J Med 2014;127:804-6. doi: 10.1016/j.amimed.2014.04.003 pmid: 24754969

33 Crowley J, Ball L, Hiddink GJ. Nutrition in medical education: a systematic review. Lancet Planet Health 2019;3:e379-89. doi: 10.1016/S2542-5196(19)30171-8 pmid: 31538623

34 Ioannidis JP. The challenge of reforming nutritional epidemiologic research. JAMA 2018:320:969-70

35 Van Horn L, Lenders CM, Pratt CA, etal. Advancing nutrition education, training, and research for medical students, residents, fellows, attending physicians, and other clinicians: building competencies and interdisciplinary coordination. Adv Nutr 2019;10:1181-200. doi: 10.1093/advances/nmz083 pmid: 31728505

36 Ramachandran D, Kite J, Vassallo AJ, etal. Food trends and popular nutrition advice online-implications for public health. Online J Public Health Inform 2018;10:

37 Mozaffarian D, Forouhi NG. Dietary guidelines and health-is nutrition science up to the task?BM 2018;360:k822. doi: 10.1136/bmi.k822 pmid: 29549076

38 Vetter ML, Herring SJ, Sood M, Shah NR, Kalet AL. What do resident physicians know about nutrition? An evaluation of attitudes, self-perceived proficiency and knowledge. / Am Coll Nutr 2008;27:287-98. doi: 10.1080/07315724.2008.10719702 pmid: 18689561

39 Hughes AM, Gregory ME, Joseph DL, etal. Saving lives: a meta-analysis of team training in healthcare. J Appl Psychol 2016;101:1266-304. doi: 10.1037/apl0000120 pmid: 27599089

40 Razavi AC, Monlezun DJ, Sapin A, etal. Multisite culinary medicine curriculum is associated with cardioprotective dietary patterns and lifestyle medicine competencies among medical trainees. Am J Lifestyle Med 2020;14:225-33. doi: 10.1177/1559827619901104. pmid: 32231488

41 Stauber Z, Razavi AC, Sarris L, Harlan TS, Monlezun DJ. Multisite medical student-led community culinary medicine classes improve patients' diets: machine learning-augmented propensity score-adjusted fixed effects cohort analysis of 1381 subjects. Am J Lifestyle Med 2019. doi: 10.1177/1559827619893602.

42 Dent E, Wright O, Hoogendijk EO, Hubbard RE. Nutritional screening and dietitian consultation rates in a geriatric evaluation and management unit. Nutr Diet 2018;75:11-6. doi: 10.1111/1747-0080.12391. pmid: 29164776

43 Broad Leib EM, Shapiro M, Chan A, et al. Doctoring our diet: policy tools to include nutrition in USUS medical training. 2019 Sept; Harvard Food Law \& Policy Clinic. Available from https://www.chlpi.org/wp-content/uploads/2013/12/Doctoring-Our-Diet_-September-2019-V2.pdf. 
44 Prescribing Gluten-Free foods in Primary Care: Guidance for Clinical Commissioning Groups frequently asked questions, NHS England, [cited 2020 May 15], available at https://www.england.nhs.uk/medicines-2/medicines-optimisation/prescribing-gluten-free-foods-in-primary-careguidance-for-ccgs-faqs/\#why-are-gf-foods-available-on-the-nhs.

45 Medi-Cal medically tailored meals pilot operating in the state of California's Medicaid program. https://www.dhcs.ca.gov/services/tt/Pages/Medically-tailored-Meals-Pilot-Program.aspx

46 Freeman WJ, Weiss AJ, Heslin KC. Statistical brief \#246: overview of US hospital stays in 2016; variation by geographic region. Agency for Healthcare Research and Quality. 2018. hcupus.ahrq.gov/reports/statbriefs/sb246-Geographic-Variation-Hospital-Stays.jsp.

47 Simmet A, Tinnemann P, Stroebele-Benschop N. The German food bank system and its users-a cross-sectional study. Int J Environ Res Public Health 2018;15:1485. doi: 10.3390/ijerph15071485 pmid: 30011832

48 Ministry for Foreign Affairs of Finland, Finnish National Agency for Education. School meals for all: investment in effective learning-case Finland. June 2019 https://www.oph.fi/sites/default/files/documents/um_casestudyfinland_schoolfeeding_june2019_netti.pdf.

49 Shei A, Costa F, Reis MG, Ko Al. The impact of Brazil's Bolsa Família conditional cash transfer program on children's health care utilization and health outcomes. BMC Int Health Hum Rights 2014;14:10. doi: 10.1186/1472-698X-14-10 pmid: 24690131

50 García-Guerra A, Neufeld LM, Bonvecchio Arenas A, etal. Closing the nutrition impact gap using program impact pathway analyses to inform the need for program modifications in Mexico's conditional cash transfer program. J Nutr 2019;149(Suppl 1):2281S-9S.

doi: 10.1093/in/nxz169 pmid: 31793648

This is an Open Access article distributed in accordance with the Creative Commons Attribution Non Commercial (CC BY-NC 4.0) license, which permits others to distribute, remix, adapt, build upon this work non-commercially, and license their derivative works on different terms, provided the original work is properly cited and the use is non-commercial. See: http://creativecommons.org/licenses/by$\mathrm{nc} / 4.0 \%$. 